GEORGETOWN SCIENTIFIC RESEARCH JOURNAL
Volume One Edition One February 2021

Determinants of Hepatocellular Carcinoma in the United States: Differences in Risk Factor and Genetic Susceptibility by Race/

\title{
Ethnicity
}

Mehwish Rafique, Dana Kristjansson, PhD. 


\title{
Determinants of Hepatocellular Carcinoma in
}

\section{the United States: Differences in Risk Factor and Genetic Susceptibility by Race/Ethnicity}

\author{
Mehwish Rafique $^{1}$ and Dana Kristjansson ${ }^{2}$ \\ ${ }^{1}$ Department of Systems Medicine, Georgetown University School of \\ Medicine, Washington DC \\ ${ }^{2}$ Department of Genetics and Bioinformatics, Norwegian Institute of Public \\ Health, Oslo, Norway
}

\section{E-mail: Mr1502@georgetown.edu}

\begin{abstract}
Background: Hepatocellular carcinoma (HCC) is one of the few cancers with an increasing incidence and mortality worldwide. This study aims to determine the contribution of known risk factors for HCC by race and ethnicity.
\end{abstract}

Methods: Data on race, ethnicity, age, and gender were obtained from National Health and Nutrition Examination Survey (NHANES). Population attributable fractions (PAFs) of risk factors were estimated using non-invasive scoring measures of Hepatitis B and C virus infection, excessive alcohol use, smoking, diabetes and emerging metabolic risk factors [non-alcoholic steatohepatitis advanced cirrhosis (NASH) and non-alcoholic fatty liver disease-advanced fibrosis (NAFLD-fib)] over a 10-year period, 1999-2002 and 2009-2012. Genetic analysis was performed using DisGenet platform by attaining the top enriched genes strongly related to HCC. Furthermore, cytoscape network was used to form a gene-disease network association.

Results: NASH-cirrhosis increased in the overall population and among all race and ethnic groups. Both liver fat accumulation and ALT levels vary among different populations; however, Hispanics have the highest prevalence of NAFLD and elevated ALT levels. Non-Hispanic (NH) blacks and Hispanics had a 3 to 4 times higher PAF for HCC than whites attributed due to chronic liver diseases, including NASHcirrhosis and NAFLD-fib. Our genetic analysis demonstrated that PNPLA3 polymorphism is strongly associated with NAFLD-fib, which appears to represent susceptibility to liver disease among the Hispanic community.

Conclusion: Hispanics and NH blacks are at a disproportionately higher risk for HCC in part due to the higher prevalence of liver disease comorbidities, including NASH-cirrhosis and NAFLD-fib. Compared 
to $\mathrm{NH}$ whites, Hispanics and NH blacks have a higher baseline risk for liver cancer due to non-metabolic factors, which may include a genetic susceptibility. Metabolic risk factors have increased and are now contributing to nearly half of $\mathrm{HCC}$ cases in the US.

Keywords: non-alcoholic fatty liver disease advanced fibrosis; hepatocellular carcinoma; non-alcoholic steatohepatitis; metabolic risk factors; non-metabolic risk factors; population attributable fraction

\section{Introduction}

Hepatocellular carcinoma (HCC) is the third most common cause of cancer related deaths worldwide. $^{1}$ In the US, HCC incidence and mortality rates are increasing at a rate of $3 \%$ per year and are distributed disproportionally among certain racial/ethnic groups. ${ }^{2}$

HCC most often occurs among individuals who have chronic liver diseases. Nonalcoholic fatty liver disease (NAFLD) and non-alcoholic steatohepatitis (NASH) are growing and becoming the leading risk factors for HCC. NAFLD-fib and its subtype NASH-cirrhosis affect approximately $30 \%$ and 5\%, respectively, of the US population. ${ }^{3}$ The major risk factors are hepatitis $\mathrm{C}$ virus $(\mathrm{HCV})$ infection, hepatitis $\mathrm{B}$ virus (HBV) infection, cigarette smoking, excessive alcohol consumption, hereditary genetic diseases, and metabolic disorders (diabetes, obesity, impaired glucose tolerance, metabolic syndrome, and non-alcoholic fatty liver disease). ${ }^{4}$ Some risk factors of metabolic diseases have been shown to be more prominent in certain ethnic groups. Data from the United States National Center for Health Statistics (2000-2006) identified chronic liver diseases as the sixth most common cause of death in the Hispanic population. ${ }^{5}$ Obesity and diabetes are highly prevalent among both Hispanic and non-Hispanic (NH) Blacks due to lifestyle choices, diet, or genetic polymorphism, which causes all racerelated genetic differences between different groups. The proportion of incident cases of heavy drinkers in the United States between 1984 and
1992 was highest among NH blacks (51\%), followed by Hispanics (43\%) and whites (32\%). There are several lines of evidence suggesting that $\mathrm{NH}$ blacks who consume alcohol have greater liver enzyme elevation than whites, which further leads to liver disease.$^{6,7}$

The prevalence of NAFLD-fib and risk of progression is higher among Hispanics than other racial and ethnic groups. ${ }^{8}$ The higher incidence of HCC among Hispanics is driven by higher levels of sugar, carbohydrates and intake of saturated fat as compared to whites. Obesity and insulin resistance, two important risk factors for the metabolic syndrome, have been found to have a positive correlation with NASH-cirrhosis in Hispanic persons only. ${ }^{5}$ Hispanics and NH blacks have also been shown to have higher HCC rates than whites. Cirrhosis rates are higher for $\mathrm{NH}$ blacks than for whites, and the highest cirrhosis mortality rates are observed among Hispanics. ${ }^{7}$ Mortality from chronic liver disease in Hispanic people in the United States is nearly 50\% higher than in $\mathrm{NH}$ white persons $(13.7$ per 100,000 in Hispanic persons vs 9.2 in $\mathrm{NH}$ whites and 7.5 in African American persons). ${ }^{5}$

In addition to known $\mathrm{HCC}$ risk factors, it is likely that access to preventive health education and early treatment may be a barrier to some racial and ethnic groups. The incidence of HCC varies by race and ethnicity primarily as a result of differences in the prevalence of major risk factors and also disparities in access to high-quality healthcare. ${ }^{9}$ Socioeconomic disadvantage, lack of health insurance, and language barriers limit access to cancer screening and treatment among $\mathrm{NH}$ blacks and Hispanics. ${ }^{10}$ It has also been found that 
Hispanics and NH blacks were less likely to be diagnosed with early-stage HCC compared with whites. $^{10}$

There is a need to understand how $\mathrm{HCC}$ risk factors contribute to $\mathrm{HCC}$ prevalence rates within racial and ethnic groups, to reduce health-care disparities. The aim of this study was to determine contribution of specific known risk factors for HCC by race and ethnicity, using a nationally representative US population. A further network study of gene specificity and HCC was conducted.

\section{Methods}

\subsection{Study Population}

The National Health and Nutrition Examination Survey (NHANES) is a biennial cross-sectional survey representative of the US civilian, non-institutionalized population. Details of the NHANES methods and sampling strategy have been described by the National Center for Health Statistics (NCHS) of the Centers for Disease Control and Prevention (CDC).${ }^{11}$ Briefly, subjects were recruited though a multistage probability sampling design, which was used to select participants representative of the civilian, non-institutionalized US population, with a sample weight assigned to each person. ${ }^{12}$ Next, each subject was interviewed and underwent a physical examination, including a blood draw. General demographic characteristics, including age, sex, race/ethnicity (non-Hispanic white, nonHispanic Black, Mexican American, other Hispanic, other race including Asian descent and multiracial (other/mixed)), and smoking behavior were collected during the Mobile Examination Center interview stage. During the examination, body measurements, including height, weight, and waist circumference $(\mathrm{cm})$ were also collected. Serum samples were obtained and analyzed for albumin ( $\mathrm{g} / \mathrm{dL})$, alanine aminotransferase (ALT, U/L), aspartate (aminotransferase (AST,
$\mathrm{U} / \mathrm{L})$, alkaline phosphatase (U/L), fasting glucose $(\mathrm{mg} / \mathrm{dL})$, fasting insulin $(\mathrm{uU} / \mathrm{mL})$, gammaglutamyl transpeptidase (GGT, U/L), platelet count $(1000$ cells $/ \mu \mathrm{L})$, total bilirubin $(\mathrm{mg} / \mathrm{dL})$, hemoglobin $\mathrm{A}_{1 \mathrm{C}}(\%)$, total cholesterol $(\mathrm{mg} / \mathrm{dL})$, high density lipoprotein (HDL), low density lipoprotein (LDL) cholesterol $(\mathrm{mg} / \mathrm{dL})$, and triglycerides $(\mathrm{mg} / \mathrm{dL})$. All participants provided informed consent. NHANES is approved by the Institutional Review Board of the CDC. Subjects who were less than 18 years old or pregnant were excluded from this analysis (Figure 1).

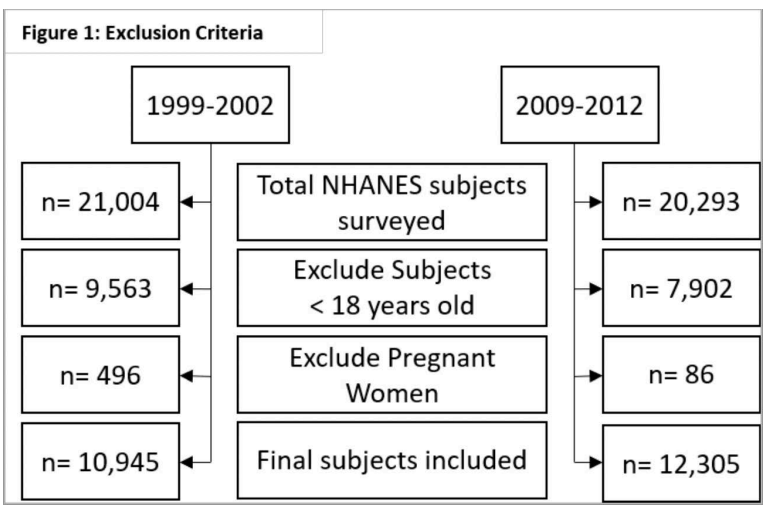

Figure 1. Exclusion Criteria

The prevalence of $\mathrm{HCC}$ risk factors was determined using interview, physical exam, and/or laboratory NHANES data. Hepatitis $\mathrm{C}$ virus was defined as having a positive hepatitis $\mathrm{C}$ virus antibody (anti-HCV) in laboratory testing. Hepatitis B viral infection was defined as having a positive surface antigen ( $\mathrm{HBsAg}$ ) on laboratory testing. Persons were identified as smokers when they reported current smoking on the NHANES questionnaire. Men who reported consuming more than 14 drinks per week and women who reported more than 7 drinks per week were defined as excessive drinkers. ${ }^{13}$

Of the metabolic risk factors, obesity was defined by BMI greater than or equal to 30 from body measurements taken on physical exam. Metabolic syndrome was defined using the 
International Diabetes Federation definition. ${ }^{14}$ Persons with any three of the following five criteria were defined as having metabolic syndrome: 1) Elevated waist circumference $(\mathrm{men}>102 \mathrm{~cm}$, women $>88 \mathrm{~cm}$ ) which was measured during physical exam; 2) Elevated triglycerides ( $>=150$ $\mathrm{mg} / \mathrm{dL}$ or currently taking prescription to lower lipids); 3) Reduced high-density lipoprotein $(<40 \mathrm{mg} / \mathrm{dL}$ for males or $<50 \mathrm{mg} / \mathrm{dl}$ for females); 4$)$ Hypertension (blood pressure measurements greater than $140 \mathrm{mg} / \mathrm{dL}$ for systolic blood pressure or greater than 90 for diastolic); or 5) Elevated fasting glucose $(\geq 100 \mathrm{mg} / \mathrm{dL})$. Diabetes was defined as having answered yes to the questionnaire question of "Have you ever been diagnosed by a physician as having diabetes?" or "Are you currently taking a blood glucose lowering medication?", and/or having a fasting glucose level greater than $126 \mathrm{mg} / \mathrm{dL}$, or having a hemoglobin $\mathrm{A}_{1 \mathrm{C}}$ level greater than 6.5\%.

Cirrhosis was defined as having an AST-toplatelet ratio index (APRI) $>2$ and any one of the following abnormal liver function tests: 1 ) elevated
ALT levels ( $>40 \mathrm{U} / \mathrm{L}$ for men or $>30 \mathrm{U} / \mathrm{L}$ for women); 2) Elevated alkaline phosphatase ( $>113$ $\mathrm{U} / \mathrm{L})$; or 3) elevated total bilirubin ( $>1.3 \mathrm{mg} / \mathrm{dL})$. NAFLD-advanced fibrosis (NAFLD-fib) was defined using three different noninvasive formulas: hepatic steatosis index (HSI), the FIB-4 index (FIB4), and the NAFLD fibrosis score (NFS).15,16 Persons who had fatty liver based on the HSI and had fibrosis based on the FIB4 and/or the NFS were defined as having NAFLD-advanced fibrosis.

$$
\begin{aligned}
& \text { HSI } \\
& =8 \times\left(\frac{A L T}{A S T}\right) \\
& +B M I[+2 \text { if Diabetes, }+2 \text { if Female }] \\
& \boldsymbol{F I B 4}=\frac{\text { Age }_{\text {Years }} \times A S T\left(\frac{U}{L}\right)}{\text { Platelet Count }\left(\frac{10^{9}}{L}\right) \times \sqrt{\operatorname{ALT}\left(\frac{U}{L}\right)}}
\end{aligned}
$$

$$
\begin{gathered}
\text { NAFLD Fibrosis Score }= \\
\frac{-1.675+0.037\left(\text { Age }_{\text {Year }}\right)+0.094(\text { BMI })+1.13(\text { Fasting Glucose })}{(\text { Diabetes }(y e s=1, n o=0))+0.99\left(\frac{A S T}{A L T}\right)-0.013\left(\text { Platelet Count }\left(\frac{10^{9}}{L}\right)-0.66\left(\text { Albumin }\left(\frac{g}{L}\right)\right)\right.}
\end{gathered}
$$

NHANES does not include genetic disorders which are risk factors for $\mathrm{HCC}$ or impaired glucose tolerance. Thus, these risk factors could not be included in our analysis.

\subsection{Statistical Analysis.}

This analysis used the required weighting procedures to account for the survey design of NHANES. Descriptive analyses were done to compare the NHANES population in 1999-2002 to the NHANES population in 2009-2012. Categorical variables were compared using $\chi^{2}$ tests. Continuous variables were compared using the Students t-test after confirming all data were normally distributed. Age-adjusted prevalence rates for $\mathrm{HCV}$ infection, $\mathrm{HBV}$ infection, smoking, excessive alcohol use, obesity, diabetes, NASHcirrhosis, and NAFLD-fib for the two four-year time periods were calculated using the projected population of the United States for the year 2000. ${ }^{17}$

To determine the predicted contribution of each risk factor towards the development of HCC, the population attributable risk was calculated. A medical literature review was done to find the relative risk (RR) of each risk factor $(\mathrm{HCV}, \mathrm{HBV}$, etc.) towards the development of HCC. The literature review was done using PubMed and with the term for each risk factor, risk, and hepatocellular carcinoma. 
Results were sorted by year of publication with US populations, and recent meta or pooled analyses preferred. The population attributable fraction (PAF) for each risk factor was then calculated using the formula developed by Levin for each sex and race group ${ }^{18}$

\section{Population Attributable Fraction$$
=\frac{\text { Risk Prevalence }\left(R R_{\text {Exposure }}-1\right)}{\text { Risk Prevalence }\left(R R_{\text {Exposure }}-1\right)+1}
$$

In determining the PAF, the risk factors were analyzed independently, without accounting for interaction between their effects.

The combined effect of all risk factors and risk factors by type (metabolic vs. non-metabolic) were calculated using the formula below:

\section{Total Attributable Fraction}

$$
=1-\prod_{i=1}^{n}\left(1-P A F_{i}\right)
$$

All statistical analyses were conducted using SAS 9.4 (Cary, NC) with $p<0.05$ considered significant. Figures were developed using GraphPad Prism version 8.0.0 for Windows, GraphPad Software, San Diego, California USA.

The top enriched pathogenic genes associated with liver diseases were analyzed using the DisGenet platform (Table 1). ${ }^{19}$ The DisGeNet database uses information of human gene-disease association (GDAs) and variant-disease association (VDAs) from expert curated repositories. The GDA score was calculated using the formula developed by DisGeNet. Scoring (gda) was used to rank the gene-disease according to their level of evidence. ${ }^{19}$
The DisGeNET Score (S) for GDAs is computed according to:

$$
\begin{aligned}
& S=C+M+I+L \\
& C= \begin{cases}0.6 \quad \text { if } N_{\text {sources }_{i}}>2 \\
0.5 \quad \text { if } N_{\text {sources }_{i}}=2 \\
0.3 \quad \text { if } N_{\text {sources }_{i}}=1 \\
0 \quad \text { otherwise }\end{cases}
\end{aligned}
$$

where:

$\mathrm{N}_{\text {sourcesi }}$ is the number of CURATED sources supporting a GDA i $\in$ CGI, CLINGEN, GENOMICS ENGLAND, CTD, PSYGENET, ORPHANET, UNIPROT

$$
M= \begin{cases}0.2 & \text { if } N_{\text {sources }_{j}}>0 \\ 0 & \text { otherwise }\end{cases}
$$

where:

$\mathrm{j} \in$ Rat, Mouse from RGD, MGD, and CTD

$$
I= \begin{cases}0.1 & \text { if } N_{\text {sources }_{k}}>0 \\ 0 & \text { otherwise }\end{cases}
$$

where:

$\mathrm{k} \in \mathrm{HPO}, \mathrm{CLINVAR}$, GWASCAT, GWASDB

$$
L= \begin{cases}0.1 & \text { if } N_{\text {pubs }}>9 \\ N_{\text {pubs }} * 0.01 & \text { if } N_{\text {pubs }}<9\end{cases}
$$

where:

$\mathrm{N}_{\text {pubs }}$ is the number of publications supporting a GDA in the sources LHGDN and BEFREE

DisGeNET uses two other metrics to facilitate the ranking of the genes associated with hepatocellular carcinoma. The Disease Specificity Index (DSI) was used, which is inversely proportional to the number of diseases associated to gene. A gene associated with multiple diseases 


\section{https://doi.org/10.48091/LKML8578}

gets a score close to zero, and a gene associated with only one disease has DSI of $1 .{ }^{18}$ It is computed according to :

$$
D S I=\frac{\log _{2}\left(\frac{N_{d}}{N_{T}}\right)}{\log _{2}\left(\frac{1}{N_{T}}\right)}
$$

where:

$\mathrm{N}_{\mathrm{d}}$ Is the number of diseases associated to the gene/variant

$\mathrm{N}_{\mathrm{T}}$ is the total number of diseases in

\section{DisGeNET}

The Disease Pleiotropy Index (DPI) was the second metric used to rank the genes. It ranges from 0 to 1 and is proportional to the number of different $(\mathrm{MeSH})$ disease classes a gene is associated with. The DPI is computed according to :

$$
D P I=\left(\frac{N_{d c}}{N_{T C}}\right) * 100
$$

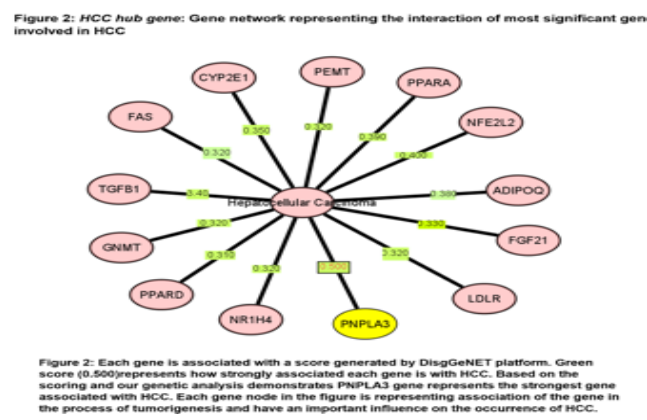

In the overall NHANES population, obesity, excessive alcohol consumption, and smoking were the most prevalent HCC risk factors in both 19992002 and 2009-2012. The prevalence of HBV, $\mathrm{HCV}$, or excessive alcohol consumption did not change over the ten-year period. All metabolic risk where:

$\mathrm{N}_{\mathrm{dc}}$ is the number of the different $\mathrm{MeSH}$ disease classes of the diseases associated to the gene/variant

$\mathrm{N}_{\text {TC }}$ is the total number of $\mathrm{MeSH}$ diseases classes in DisGeNET.

Furthermore, cytoscape network was used to form a gene-disease association to visualize interaction among different genes (Figure 2). Cytoscape is an open-source platform for visualizing molecular interactions. ${ }^{20}$

\section{Results}

There were 10,945 individuals in the 19992002 sample and 12,305 in the 2009-2012 sample (Figure 1). The groups did not differ among the distribution of sex, age, or race/ethnicity. The mean age at screening was 45.4 (1999-2002) and 46.4 (2009-2012) and $50.7 \%$ of the participants were female (Table 2).

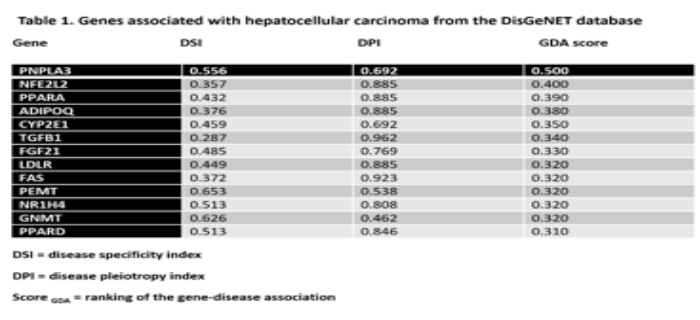

factors (obesity, diabetes, NAFLD-fib, NASHcirrhosis) increased over the ten years of study in the overall population (Table 2). Concurrently, BMI, waist circumference, fasting glucose levels, and triglyceride levels all increased between the two time periods (all $\mathrm{p}<0.001$, Supplemental Table 1). 
Table 2. Participant Characteristics

Characteristics

Survey Period

\begin{tabular}{|c|c|c|c|c|}
\hline & & 1999-2002 & 2009-2012 & p-value \\
\hline Demographics & Gender & & & 0.15 \\
\hline \multirow[t]{11}{*}{$\mathrm{N} \%$} & Male & $5395(49.3)$ & $6072(49.4)$ & \\
\hline & Female & $5550(50.7)$ & $6233(50.7)$ & \\
\hline & $\begin{array}{l}\text { Age at Screening } \\
\text { (years) } *\end{array}$ & $45.4(0.33)$ & $46.4(0.51)$ & 0.22 \\
\hline & Race/Ethnicity & & & 0.19 \\
\hline & Non-Hispanic White & $5132(46.9)$ & $5158(47.9)$ & \\
\hline & Non-Hispanic Black & $2163(19.8)$ & $2734(22.2)$ & \\
\hline & Hispanic & $3273(29.9)$ & $3065(24.9)$ & \\
\hline & Mixed/Other & $377(3.44)$ & $1348(10.9)$ & \\
\hline & Smoking Status & & & $<0.001$ \\
\hline & Current Smoker & $2125(24.5)$ & $2441(20.3)$ & \\
\hline & Former Smoker & $2625(26.8)$ & $2768(23.7)$ & - \\
\hline Disease Prevalence & Hepatitis C Virus & $2.0(54)$ & $1.6(48)$ & 0.37 \\
\hline \multirow{7}{*}{$\begin{array}{l}\text { \% Prevalence } \\
\text { (Population } \\
\text { Attributable Fraction) }\end{array}$} & Hepatitis B Virus & $0.2(5)$ & $0.4(7)$ & 0.67 \\
\hline & Excessive Alcohol & $23.8(12)$ & $21.6(12)$ & 0.83 \\
\hline & Current Smokers & $24.5(12)$ & $20.3(10)$ & $<0 / 001$ \\
\hline & Diabetes & $9.8(9)$ & $11.8(11)$ & $<0.001$ \\
\hline & Obesity & $29.9(25)$ & $35.2(28)$ & $<0.001$ \\
\hline & NASH-Cirrhosis & $0.07(3)$ & $0.2(6)$ & 0.02 \\
\hline & NAFLD-fib & $1.5(1.4)$ & $4.0(3.7)$ & $<0.001$ \\
\hline
\end{tabular}

$N A S H=$ non-alcoholic steatohepatitis; $N A F L D-f i b=$ non=alcoholic fatty liver disease advanced fibrosis

\begin{tabular}{|c|c|c|c|c|c|}
\hline \multirow[t]{2}{*}{ Metabolic Measures } & \multicolumn{2}{|c|}{ 1999-2002 } & \multicolumn{3}{|c|}{ 009-2012 } \\
\hline & mean & s.e.m. & mean & s.e.m & p-value \\
\hline $\mathrm{BMI}\left(\mathrm{kg} / \mathrm{m}^{\wedge} 2\right)$ & 27.9 & 0.13 & 28.6 & 0.49 & $<0.001$ \\
\hline $\begin{array}{l}\text { Waist Circumference } \\
(\mathrm{cm})\end{array}$ & 95.3 & 0.16 & 98.1 & 0.16 & $<0.001$ \\
\hline $\begin{array}{l}\text { Mean Systolic Pressure } \\
(\mathrm{mmHg})\end{array}$ & 123.2 & 0.42 & 121.1 & 0.41 & $<0.001$ \\
\hline Fasting Glucose $(\mathrm{mg} / \mathrm{dL})$ & 104.3 & 0.55 & 107.1 & 0.45 & $<0.001$ \\
\hline Fasting Insulin (uU.mL) & 13.0 & 0.34 & 13.8 & 0.26 & 0.17 \\
\hline $\begin{array}{l}\text { Cholesterol, total } \\
(\mathrm{mg} / \mathrm{DI})\end{array}$ & 197.9 & 0.78 & 193.1 & 0.73 & $<0.001$ \\
\hline HDL-cholesterol (mg/DI) & 51.2 & 0.36 & 52.9 & 0.59 & $<0.001$ \\
\hline LDL-cholesterol (mg/DI) & 122.4 & 0.96 & 115.0 & 0.71 & $<0.001$ \\
\hline Triglycerides & 144.6 & 2.62 & 150.4 & 2,24 & $>0.001$ \\
\hline $\begin{array}{l}\text { Fasting Glucose }>100 \\
(\%)\end{array}$ & 16.0 & 0.56 & 19.5 & 0.63 & $<0.001$ \\
\hline Albumin(g/dL) & 4.37 & 0.01 & 4.29 & 0.01 & $<0.001$ \\
\hline ALT (U/L) & 25.9 & 0.42 & 25.4 & 0.23 & 0.44 \\
\hline AST (U/L) & 24.4 & 0.22 & 25.9 & 0.16 & $<0.001$ \\
\hline GGT (U/L) & 84.9 & 0.49 & 69.0 & 0.24 & $<0.001$ \\
\hline Platelet count (SI) & 268.5 & 1.39 & 239.0 & 1.13 & $<0.001$ \\
\hline Total Bilirubin (mg/dL) & 0.58 & 0.01 & 0.74 & 0.01 & $<0.001$ \\
\hline GGT (U/L) & 30.3 & 0.46 & 26.6 & 0.50 & $<0.001$ \\
\hline Iron (ug/dL) & 87.2 & 0.39 & 84.2 & 0.34 & $<0.001$ \\
\hline
\end{tabular}

s.e.m. = standard error of the mean; ALT= Alanine aminotransferase; AST=Aspartate aminotransferase; GGT= GammaGlutymyl Transpetidase;

Both NASH-cirrhosis and NAFLD-fib increased in prevalence between 1999-2002 and 2009-2012. The prevalence rates of NAFLD-fib increased from 1999-2002 (1.53\%) to 2009-2012 (4.0\%) $(\mathrm{p}<0.001)$, while NASHcirrhosis 
increased from $0.07 \%$ to $0.20 \%, \mathrm{p}=0.03)$. This represents a $164 \%$ increase for NAFLD-fib and $186 \%$ increase for NASH-cirrhosis overall. The largest increase of NAFLD-fib occurred among the Hispanic population (a 296\% increase, $\mathrm{p}<0.001)$ (Table 3).

Table 3A and B: Hepatocellular Carcinoma Risk Factor Prevalence and PAF by Sex and Race/Ethnicity

Table3a: Hepatocellular Carcinoma Risk Factor Prevalence and PAF Stratified by Sex and Race/Ethnicity Subgroup (1999-2002)

\begin{tabular}{|c|c|c|c|c|c|c|c|c|c|c|c|c|c|c|c|c|c|}
\hline & & \multicolumn{2}{|c|}{ HCV } & \multicolumn{2}{|l|}{ HBV } & \multicolumn{2}{|c|}{ Excessive alcohol } & \multicolumn{2}{|c|}{ Current smoker } & \multicolumn{2}{|c|}{ Diabetes } & \multicolumn{2}{|c|}{ Obesty } & \multicolumn{2}{|c|}{ NASH cirrhosis } & \multicolumn{2}{|c|}{ NAFLD-fib } \\
\hline & & Pred $(x)$ & PAF(X) & Prevel(x) & PNF(X) & Presed $(x)$ & PAF(X) & Prest $(x)$ & PAF(X) & $\operatorname{Prex}(\mathbf{S})$ & $P N F(x)$ & Previn: & PAF(X) & Previ(x) & PAF(X) & Prevl:s] & PAF (א) \\
\hline Overall & $\begin{array}{l}\text { Total } \\
\text { M } \\
\text { F }\end{array}$ & $\begin{array}{l}2.0 \\
2.6 \\
1.4\end{array}$ & $\begin{array}{l}54 \\
52 \\
53\end{array}$ & $\begin{array}{l}0.2 \\
0.3 \\
0.2\end{array}$ & $\begin{array}{l}5 \\
7 \\
2\end{array}$ & $\begin{array}{l}23.8 \\
22.3 \\
26.9\end{array}$ & $\begin{array}{l}12 \\
11 \\
13\end{array}$ & $\begin{array}{l}24.5 \\
26.9 \\
22.1\end{array}$ & $\begin{array}{l}12 \\
13 \\
11\end{array}$ & $\begin{array}{l}9.8 \\
11.2 \\
8.5\end{array}$ & $\begin{array}{l}9 \\
10 \\
8\end{array}$ & $\begin{array}{l}29.9 \\
26.7 \\
32.8\end{array}$ & $\begin{array}{l}25 \\
23 \\
27\end{array}$ & $\begin{array}{l}0.07 \\
0.05 \\
0.09\end{array}$ & $\begin{array}{l}3 \\
2 \\
4\end{array}$ & $\begin{array}{l}1.5 \\
1.5 \\
1.6\end{array}$ & $\begin{array}{l}1 \\
1 \\
1\end{array}$ \\
\hline $\begin{array}{l}\text { NH White } \\
N=5132\end{array}$ & $\begin{array}{l}\text { Total } \\
\text { M } \\
\text { F }\end{array}$ & $\begin{array}{l}1.9 \\
2.4 \\
1.3\end{array}$ & $\begin{array}{l}53 \\
60 \\
34\end{array}$ & $\begin{array}{l}0.1 \\
0.0 \\
0.1\end{array}$ & $\begin{array}{l}1 \\
0 \\
0\end{array}$ & $\begin{array}{l}24.5 \\
24.1 \\
25.3\end{array}$ & $\begin{array}{l}12 \\
12 \\
13\end{array}$ & $\begin{array}{l}25.0 \\
26.1 \\
24.0\end{array}$ & $\begin{array}{l}12 \\
13 \\
12\end{array}$ & $\begin{array}{l}8.4 \\
10.7 \\
6.1\end{array}$ & $\begin{array}{l}8 \\
10 \\
6\end{array}$ & $\begin{array}{l}28.7 \\
27.2 \\
30.1\end{array}$ & $\begin{array}{l}25 \\
24 \\
25\end{array}$ & $\begin{array}{l}0.08 \\
0.04 \\
0.13\end{array}$ & $\begin{array}{l}4 \\
2 \\
6\end{array}$ & $\begin{array}{l}1.3 \\
1.1 \\
1.4\end{array}$ & $\begin{array}{l}1 \\
1 \\
1\end{array}$ \\
\hline $\begin{array}{l}\text { NH Black } \\
N=2163\end{array}$ & $\begin{array}{l}\text { Total } \\
\text { M } \\
\text { F }\end{array}$ & $\begin{array}{l}4.1 \\
6.0 \\
2.6\end{array}$ & $\begin{array}{l}70 \\
78 \\
62\end{array}$ & $\begin{array}{l}0.8 \\
0.8 \\
0.7\end{array}$ & $\begin{array}{l}19 \\
10 \\
3\end{array}$ & $\begin{array}{l}22.9 \\
18.8 \\
30.9\end{array}$ & $\begin{array}{l}11 \\
9 \\
14\end{array}$ & $\begin{array}{l}26.5 \\
33.9 \\
20.5\end{array}$ & $\begin{array}{l}13 \\
16 \\
10\end{array}$ & $\begin{array}{l}14.2 \\
10.6 \\
16.8\end{array}$ & $\begin{array}{l}13 \\
10 \\
15\end{array}$ & $\begin{array}{l}38.9 \\
26.8 \\
48.7\end{array}$ & $\begin{array}{l}31 \\
23 \\
35\end{array}$ & $\begin{array}{l}0 \\
0 \\
0\end{array}$ & $\begin{array}{l}0 \\
0 \\
0\end{array}$ & $\begin{array}{l}1.9 \\
1.2 \\
2.5\end{array}$ & $\begin{array}{l}1 \\
1 \\
1\end{array}$ \\
\hline $\begin{array}{l}\text { Hispanic } \\
\mathrm{N}=3273\end{array}$ & $\begin{array}{l}\text { Total } \\
\text { M } \\
\text { F }\end{array}$ & $\begin{array}{l}2.0 \\
2.0 \\
1.8\end{array}$ & $\begin{array}{l}44 \\
53 \\
52\end{array}$ & $\begin{array}{l}0.0 \\
0.1 \\
0.0\end{array}$ & $\begin{array}{l}0 \\
2 \\
0\end{array}$ & $\begin{array}{l}22.3 \\
22.1 \\
24.1\end{array}$ & $\begin{array}{l}10 \\
10 \\
9\end{array}$ & $\begin{array}{l}22.9 \\
27.2 \\
18.9\end{array}$ & $\begin{array}{l}11 \\
13 \\
9\end{array}$ & $\begin{array}{l}14.1 \\
13.5 \\
13.9\end{array}$ & $\begin{array}{l}12 \\
12 \\
12\end{array}$ & $\begin{array}{l}30.1 \\
23.1 \\
36.5\end{array}$ & $\begin{array}{l}25 \\
21 \\
29\end{array}$ & $\begin{array}{l}0.10 \\
0.23 \\
0\end{array}$ & $\begin{array}{l}4 \\
9 \\
0\end{array}$ & $\begin{array}{l}1.3 \\
1.2 \\
1.3\end{array}$ & $\begin{array}{l}1 \\
1 \\
1\end{array}$ \\
\hline $\begin{array}{l}\text { Other/Mixed } \\
N=377\end{array}$ & $\begin{array}{l}\text { Total } \\
\text { M } \\
\text { F }\end{array}$ & $\begin{array}{l}1.1 \\
1.4 \\
1.0\end{array}$ & $\begin{array}{l}40 \\
35 \\
36\end{array}$ & $\begin{array}{l}2.3 \\
3.6 \\
0.9\end{array}$ & $\begin{array}{l}8 \\
5 \\
21\end{array}$ & $\begin{array}{l}28.4 \\
27.6 \\
28.2\end{array}$ & $\begin{array}{l}13 \\
12 \\
16\end{array}$ & $\begin{array}{l}22.3 \\
31.4 \\
14.3\end{array}$ & $\begin{array}{l}11 \\
15 \\
7\end{array}$ & $\begin{array}{l}17.9 \\
18.3 \\
17.5\end{array}$ & $\begin{array}{l}15 \\
16 \\
15\end{array}$ & $\begin{array}{l}20.3 \\
22.3 \\
19.2\end{array}$ & $\begin{array}{l}19 \\
20 \\
18\end{array}$ & $\begin{array}{l}0 \\
0 \\
0\end{array}$ & $\begin{array}{l}0 \\
0 \\
0\end{array}$ & $\begin{array}{l}0.8 \\
0.7 \\
0.9\end{array}$ & $\begin{array}{l}1 \\
1 \\
1\end{array}$ \\
\hline
\end{tabular}

\begin{tabular}{|c|c|c|c|c|c|c|c|c|c|c|c|c|c|c|c|c|c|}
\hline \multirow[b]{3}{*}{ Overall } & \multirow{2}{*}{\multicolumn{2}{|c|}{$\begin{array}{c}\text { HCV } \\
\operatorname{Prer}\left(x_{0}\right) \text { PNF }\left(x_{0}\right)\end{array}$}} & \multicolumn{2}{|c|}{ HBV } & \multicolumn{2}{|c|}{ Excessive Alcohol C } & \multicolumn{2}{|c|}{ Current Smokers } & \multirow{2}{*}{$\begin{array}{l}\text { Diabotes } \\
\text { Pren(x) }\end{array}$} & \multicolumn{2}{|c|}{ obesity } & \multicolumn{2}{|c|}{ NASH cirthosis } & \multicolumn{2}{|c|}{ NAFLD-fib } & \multirow{2}{*}{\multicolumn{2}{|c|}{$\operatorname{PAE}(\mathbf{X})$}} \\
\hline & & & Prevls & a PNF( $\left(x_{0}\right)$ & & $P N(X)$ & $\operatorname{Prev}(\%)$ & PAF(x) & & PNF(X) & $\operatorname{Prev}(\mathbf{x})$ & $P N(x)$ & Prevl(x) & $\operatorname{PAF}(x) P$ & $\operatorname{rex}(x)$ & & \\
\hline & $\begin{array}{l}\text { Total } \\
\text { M } \\
\text { F }\end{array}$ & $\begin{array}{l}1.6 \\
2.2 \\
1.1\end{array}$ & $\begin{array}{l}48 \\
47 \\
46\end{array}$ & $\begin{array}{l}0.4 \\
0.4 \\
0.3\end{array}$ & $\begin{array}{l}7 \\
10 \\
4\end{array}$ & $\begin{array}{l}21.6 \\
27.4 \\
23.7\end{array}$ & $\begin{array}{l}12 \\
11 \\
13\end{array}$ & $\begin{array}{l}20.3 \\
22.7 \\
18.0\end{array}$ & $\begin{array}{l}10 \\
11 \\
9\end{array}$ & $\begin{array}{l}11.8 \\
13.7 \\
10.1\end{array}$ & $\begin{array}{l}11 \\
12 \\
9\end{array}$ & $\begin{array}{l}35.2 \\
34.3 \\
36.0\end{array}$ & $\begin{array}{l}28 \\
28 \\
29\end{array}$ & $\begin{array}{l}0.20 \\
0.37 \\
0.06\end{array}$ & $\begin{array}{l}8 \\
14 \\
3\end{array}$ & $\begin{array}{l}4.0 \\
4.1 \\
4.0\end{array}$ & $\begin{array}{l}4 \\
4 \\
4\end{array}$ \\
\hline $\begin{array}{l}\text { NH White } \\
N=5158\end{array}$ & $\begin{array}{l}\text { Total } \\
\text { M } \\
\text { F }\end{array}$ & $\begin{array}{l}1.6 \\
2.1 \\
1.2\end{array}$ & $\begin{array}{l}49 \\
56 \\
31\end{array}$ & $\begin{array}{l}0.1 \\
0.2 \\
0.0\end{array}$ & $\begin{array}{l}2 \\
1 \\
0\end{array}$ & $\begin{array}{l}21.3 \\
27.1 \\
26.6\end{array}$ & $\begin{array}{l}12 \\
11 \\
13\end{array}$ & $\begin{array}{l}21.1 \\
21.9 \\
20.3\end{array}$ & $\begin{array}{l}10 \\
11 \\
10\end{array}$ & $\begin{array}{l}9.3 \\
11.5 \\
7.4\end{array}$ & $\begin{array}{l}9 \\
10 \\
7\end{array}$ & $\begin{array}{l}33.2 \\
34.0 \\
32.4\end{array}$ & $\begin{array}{l}27 \\
28 \\
27\end{array}$ & $\begin{array}{l}0.19 \\
0.43 \\
0\end{array}$ & $\begin{array}{l}8 \\
16 \\
0\end{array}$ & $\begin{array}{l}3.5 \\
3.6 \\
3.4\end{array}$ & $\begin{array}{l}3 \\
3 \\
3\end{array}$ \\
\hline $\begin{array}{l}\text { NH Black } \\
N=2734\end{array}$ & $\begin{array}{l}\text { Total } \\
\text { M } \\
\text { F }\end{array}$ & $\begin{array}{l}2.5 \\
3.3 \\
2.0\end{array}$ & $\begin{array}{l}59 \\
66 \\
55\end{array}$ & $\begin{array}{l}0.9 \\
1.2 \\
0.7\end{array}$ & $\begin{array}{l}21 \\
14 \\
2\end{array}$ & $\begin{array}{l}23.1 \\
31.8 \\
23.7\end{array}$ & $\begin{array}{l}12 \\
11 \\
14\end{array}$ & $\begin{array}{l}24.4 \\
30.0 \\
20.1\end{array}$ & $\begin{array}{l}10 \\
11 \\
9\end{array}$ & $\begin{array}{l}18.1 \\
18.3 \\
18.0\end{array}$ & $\begin{array}{l}15 \\
16 \\
15\end{array}$ & $\begin{array}{l}48.8 \\
38.2 \\
57.2\end{array}$ & $\begin{array}{l}36 \\
30 \\
39\end{array}$ & $\begin{array}{l}0.21 \\
0.00 \\
0.41\end{array}$ & $\begin{array}{l}8 \\
0 \\
15\end{array}$ & $\begin{array}{l}4.8 \\
4.5 \\
5.1\end{array}$ & $\begin{array}{l}4 \\
4 \\
5\end{array}$ \\
\hline $\begin{array}{l}\text { Hispanic } \\
N=3065\end{array}$ & $\begin{array}{l}\text { Total } \\
\text { M } \\
\text { F }\end{array}$ & $\begin{array}{l}1.4 \\
2.1 \\
0.6\end{array}$ & $\begin{array}{l}35 \\
55 \\
27\end{array}$ & $\begin{array}{l}0.1 \\
0.1 \\
0.2\end{array}$ & $\begin{array}{l}0 \\
3 \\
2\end{array}$ & $\begin{array}{l}23.1 \\
25.3 \\
22.1\end{array}$ & $\begin{array}{l}11 \\
11 \\
12\end{array}$ & $\begin{array}{l}17.0 \\
23.1 \\
10.7\end{array}$ & $\begin{array}{l}10 \\
11 \\
10\end{array}$ & $\begin{array}{l}18.9 \\
20.4 \\
17.4\end{array}$ & $\begin{array}{l}16 \\
17 \\
15\end{array}$ & $\begin{array}{l}40.8 \\
38.4 \\
43.2\end{array}$ & $\begin{array}{l}32 \\
30 \\
33\end{array}$ & $\begin{array}{l}0.23 \\
0.29 \\
0.17\end{array}$ & $\begin{array}{l}9 \\
11 \\
7\end{array}$ & $\begin{array}{l}4.0 \\
3.4 \\
4.5\end{array}$ & $\begin{array}{l}4 \\
3 \\
4\end{array}$ \\
\hline $\begin{array}{l}\text { Other/Mixe } \\
d \\
N=1348\end{array}$ & $\begin{array}{l}\text { Total } \\
\text { M } \\
\text { F }\end{array}$ & $\begin{array}{l}0.8 \\
1.8 \\
0.0\end{array}$ & $\begin{array}{l}34 \\
41 \\
0\end{array}$ & $\begin{array}{l}2.4 \\
2.5 \\
2.3\end{array}$ & $\begin{array}{l}9 \\
4 \\
41\end{array}$ & $\begin{array}{l}20.3 \\
19.4 \\
19.2\end{array}$ & $\begin{array}{l}10 \\
9 \\
10\end{array}$ & $\begin{array}{l}17.1 \\
22.3 \\
12.5\end{array}$ & $\begin{array}{l}12 \\
14 \\
10\end{array}$ & $\begin{array}{l}17.2 \\
19.8 \\
15.2\end{array}$ & $\begin{array}{l}15 \\
17 \\
13\end{array}$ & $\begin{array}{l}19.8 \\
21.1 \\
18.5\end{array}$ & $\begin{array}{l}18 \\
19 \\
17\end{array}$ & $\begin{array}{l}0.10 \\
0.23 \\
0\end{array}$ & $\begin{array}{l}4 \\
9 \\
0\end{array}$ & $\begin{array}{l}0.9 \\
0.9 \\
0.9\end{array}$ & $\begin{array}{l}1 \\
1 \\
1\end{array}$ \\
\hline
\end{tabular}

Prev=prevalence (\%); PAF= Population Attributable Fraction (\%); M=males; F=Females; SEM=Standard error or the mean; $\mathrm{HCV}=$ Hepatitis $C$ Viral infection; $H B V=$ Hepatitis $B$ viral Infection; NASH=Non-alcoholic steatohepatitis; NAFLD-

The risk factors to decrease over the ten-year period were the number of current smokers (prevalence $24.5 \%$ to $20.3 \%, \mathrm{p}=<0.001$ ) and access alcohol. There were no temporal changes in the prevalence of excessive drinking behaviors overall, which remained between $23.8 \%$ and $21.6 \%$ ( $\mathrm{p}=$ 0.83, Table 2).

\subsection{Population Attributable Fractions}

Overall, 77.9\% of HCC cases from 1999-2002 and $76.2 \%$ of HCC cases from 2009-2012 could be attributed to the risk factors analyzed in this study. In the overall population, metabolic risk factors (diabetes, obesity, NAFLD-fib, NASHcirrhosis) composed 35\% of HCC cases in 1999- 
2002 and increased to accounted for $42 \%$ of HCC cases in 2009-2012. Synchronously, nonmetabolic risk factors ( $\mathrm{HBV}, \mathrm{HCV}$, excessive alcohol use, current smokers) decreased from a PAF of $66 \%$ in $1999-2002$ to $59 \%$ in $2009-2012$ (Table 4). Other/mixed category was removed from the study because it was not statistically significant enough to make conclusions about the $\mathrm{PAF}$ due to low sample size. Among both metabolic and non-metabolic risk factors, $\mathrm{HCV}$ was the single highest attributable cause of HCC in both time periods; $54.1 \%$ of $\mathrm{HCC}$ cases were estimated to be attributable to $\mathrm{HCV}$ in 1999-2002 and $48.4 \%$ in $2009-2012$.

Table 4. PAF by Risk Category Stratified by Sex and Race/Ethnicity

Table 4A: PAF by Risk Category Stratified by Sex and Race/Ethnicity 1999-2002

\begin{tabular}{|l|l|l|l|l|l|}
\multicolumn{9}{c}{} & \multicolumn{2}{c}{ Metabolic } & Non-Metabolic Unknown & Total \\
\hline Overall & Total & $\mathbf{3 4 . 9}$ & $\mathbf{6 6 . 0}$ & $\mathbf{2 2 . 1}$ & $\mathbf{7 7 . 9}$ \\
& M & $\mathbf{3 3 . 4}$ & $\mathbf{6 5 . 4}$ & $\mathbf{2 3 . 1}$ & $\mathbf{7 6 . 9}$ \\
& F & $\mathbf{3 6 . 4}$ & $\mathbf{6 4 . 6}$ & $\mathbf{2 2 . 5}$ & $\mathbf{7 7 . 5}$ \\
\hline NH & Total & 13.1 & 63.7 & 31.5 & 68.5 \\
White & M & 32.8 & 69.3 & 20.6 & 79.4 \\
& F & 34.5 & 49.0 & 33.4 & 66.6 \\
\hline NH Black & Total & 40.4 & 81.1 & 11.3 & 88.7 \\
& M & 31.5 & 85.0 & 10.3 & 89.7 \\
& F & 46.1 & 71.3 & 15.5 & 84.5 \\
\hline Hispanics & Total & 38.2 & 54.7 & 28.0 & 72.0 \\
& M & 37.3 & 64.1 & 22.5 & 77.5 \\
& F & 38.7 & 60.1 & 24.5 & 75.5 \\
\hline
\end{tabular}

\begin{tabular}{|c|c|c|c|c|c|}
\hline & & Metabolic & Non-Metabolic & Unknown & Total \\
\hline Overall & $\begin{array}{l}\text { Total } \\
\text { M } \\
\text { F }\end{array}$ & $\begin{array}{l}42.0 \\
46.3 \\
37.8\end{array}$ & $\begin{array}{l}59.0 \\
60.9 \\
61.7\end{array}$ & $\begin{array}{l}23.8 \\
21.0 \\
23.8\end{array}$ & $\begin{array}{l}76.2 \\
79.0 \\
76.2\end{array}$ \\
\hline NH White & $\begin{array}{l}\text { Total } \\
\text { M } \\
\text { F }\end{array}$ & $\begin{array}{l}39.5 \\
45.1 \\
34.0\end{array}$ & $\begin{array}{l}61.0 \\
65.7 \\
46.7\end{array}$ & $\begin{array}{l}23.6 \\
18.8 \\
35.2\end{array}$ & $\begin{array}{l}76.4 \\
81.2 \\
64.8\end{array}$ \\
\hline NH Black & $\begin{array}{l}\text { Total } \\
\text { M } \\
\mathrm{F}\end{array}$ & $\begin{array}{l}47.9 \\
43.5 \\
51.0\end{array}$ & $\begin{array}{l}67.6 \\
78.9 \\
69.6\end{array}$ & $\begin{array}{l}16.9 \\
11.9 \\
14.9\end{array}$ & $\begin{array}{l}83.1 \\
88.1 \\
85.1\end{array}$ \\
\hline Hispanics & $\begin{array}{l}\text { Total } \\
\text { M } \\
\text { F }\end{array}$ & $\begin{array}{l}47.6 \\
49.5 \\
45.1\end{array}$ & $\begin{array}{l}49.7 \\
64.0 \\
43.8\end{array}$ & $\begin{array}{l}26.4 \\
18.2 \\
30.8\end{array}$ & $\begin{array}{l}73.6 \\
81.8 \\
69.2\end{array}$ \\
\hline
\end{tabular}

$M=$ males; F=Females; Metabolic Diseases = Diabetes, Obesity, Nonalcoholic steatohepatitis, Non-alcoholic fatty liver diseaseadvanced fibrosis; Non-Metabolic Diseases = Hepatitis B, Hepatitis C, Excessive alcohol use, Current smokers

Differences in PAF magnitudes were observed among race/ethnic subgroups. The highest $\mathrm{HCV}$ PAF was observed among non-Hispanic Blacks (59\% in 2009-2012), while the lowest was among other/mixed (34\% in 2009-2012). These values did not significantly decrease over the ten-year period. Non-Hispanic Blacks had the highest obesity PAF (31\% in 2009-2012 compared to $25 \%$ in the overall population), which decreased from $36 \%$ in 1999-2002.
NASH-cirrhosis or NAFLD-fib could be attributed to, at most, $8 \%$ of HCC cases; these liver diseases had the largest fold change of any risk factor over the study period. Specifically, NAFLD-fib had the greatest fold increase of any risk factor studied, increasing 2.6-fold. NASH-cirrhosis had the second greatest $\mathrm{PAF}$, increasing to a similar degree of 2.4-fold. NASH-cirrhosis 
increased from $3.8 \%$ of cases to $5.8 \%$ and NAFLD-fib increased from $1.4 \%$ to $3.7 \%$ $(\mathrm{p}=0.022$ and $\mathrm{p}<0.001$, respectively, Figure 3$)$.

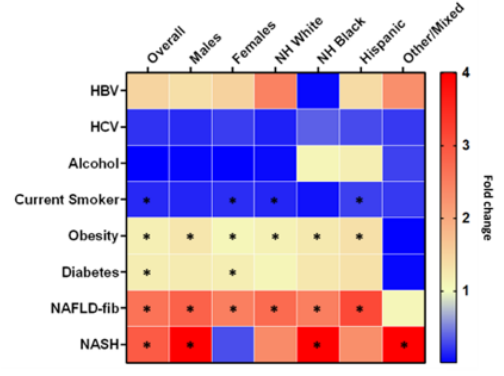

$H C V=$ Hepatitis C Viral infection; $H B V=$ Hepatitis B viral Infection; NASH=Non-alcoholic steatohepatitis; NAFLD-fib=Nonalcoholic Fatty liver disease advanced fibrosis

" indicates significant change over time ( $p<0.05$ for Chi-sq test)

Figure 3: Fold Change in Hepatocellular Carcinoma Risk Factor Population Attributablr Fractions 1999-2002 and 2009-2012

Stratification by race/ethnicity showed that obesity and NAFLD-fib increased across all groups. The largest increase was seen among Hispanics with a $208 \%$ increase in NAFLD-fib. $\mathrm{NASH}$-cirrhosis was more common among men than women in 2009-2012 (relative risk = 6.2). The largest increase in NASH-cirrhosis was among non-Hispanic Blacks, increasing from PAF of $0 \%$ in 1999-2001 to $8 \%$ in 2009-2012. NASHcirrhosis increased significantly, accounting for 6.3\% of HCC cases in 2009-2012 (Table 3, Figure $3)$.

\subsubsection{Genetic Analysis.}

There are differences in HCC outcomes between different ethnic groups. The gene PNPLA3 was shown to play a major role in the development of liver disease such as NAFLD-fib and NASH-cirrhosis. PNPLA3 represents a GDA score of 5.00(figure 2). The specificity for HCC was 0.556 , and the association of the PNPLA3 with HCC specifically is 0.692 . This is based on an evidence score of 0.500 , which was calculated from DisGenet . PNPLA3 gene represents a cytosine to guanine substitution, resulting in an isoleucine to methionine switch at codon 148 and individuals with the $G$ allele have a higher hepatic triglyceride level and elevated serum of ALT. ${ }^{21}$ Our study found a total of 13 pathogenic genes from DisGeNET platfrom with a DSI , DPI and a GDA score based on DisGeNet ranking system. Our analysis represents high frequency of PNPLA3 gene among Hispanic groups. Furthermore, genediet interaction plays a vital role in the pathogenesis of liver cancer in Hispanics.

Figure 2 represents the 13 gene strongly associated with HCC. Based on the GDA score from DisGeNET, PNPLA3 (0.500) holds a strong association with increased risk of HCC. The GDA score of PNPLA3 (0.500) is the highest as compared to other genes in list. The lowest GDA score based on DisGeNET ranking is PPARD. Genes LDLR, FAS, PEMT, NR1H4, GNMT represents the same GDA score of 0.320 which means they all are equally associated in the development of HCC.

\section{Discussion}

This study attributed nearly $80 \%$ of HCC cases in 1999-2001 and 2009-2012 to eight known risk factors in a large nationally representative sample of the U.S. population. Metabolic risk factors are now contributing to nearly half of $\mathrm{HCC}$ cases in the US. Metabolic diseases (diabetes, obesity, NASH-cirrhosis, and NAFLD-advanced fibrosis) increased from contributing an estimated $35 \%$ of HCC burden in 1999-2002 to 42\% in 2009-2012. Concordantly, non-metabolic risk factors decreased from $66 \%$ to $59 \%$ of total HCC burden over the same period. Stratification by race/ethnicity showed a similar shift across all groups. The HCC risk factor prevalence rates of obesity and NAFLD-fib increased for all groups and were particularly high among Hispanics and non-Hispanic Blacks. A genetic variant in $P N P L A 3$ was identified as strongly associated with HCC. 
These findings highlight vulnerabilities within certain racial and ethnic groups within one country's population of $\mathrm{HCC}$, which is increasing in the US as well as worldwide. ${ }^{25-28}$ As the obesity epidemic had increased from 1999 until 2012, metabolic risk factors (NAFLD-fib and NASHcirrhosis) became contributers to HCC development in the US more so than nonmetabolic risk factors ( $\mathrm{HBV}, \mathrm{HCV})$, excessive alcohol use, and smoking. ${ }^{22,23}$

It is likely that there is an interplay between lack of access to health care, racial disparities, and genetics leading the whole pathogenesis and playing a major role in the development of liver disease that eventually forms into HCC. Specific genetic contributions may help explain differences observed in PAFs for HCC between race/ethnic groups. ${ }^{29}$ While insulin resistance likely plays a role in its pathogenesis, oxidative injury and inflammatory reactions could be influenced by genetics. A study performed in the US among Hispanic, $\mathrm{NH}$ black and $\mathrm{NH}$ white individuals identified the variant, rs $73809,(148 \mathrm{M})$ in patatinlike phospholipase domain-containing protein 3 (PNPLA3) as a predictor for hepatic fat content. ${ }^{30}$ The study confirmed that patients with NAFLDfib who carry an allele of the gene (rs73809) PNPLA3 have an increased risk of developing advanced diseases, including NASH-cirrhosis. Risk allele (rs73809) was the main common genetic determinant of hepatic fat content and of progressive NAFLD-fib, and this allele was mostly observed among Hispanic groups. It is not clear as to why this allele is increased among Hispanics. The variant has been reported to manifest in early life among Hispanic adolescents, ${ }^{31}$ as well was having a prevalence of $80 \%$ in a single center study in Mexico. ${ }^{32}$ This is consistent with the current study's findings showing higher burdens of NAFLD-fib among Hispanics. Overall, evolving knowledge in genetics along with epidemiological studies focused on race/ethnic backgrounds may help identify patients at higher risks for HCC. ${ }^{33}$ This study demonstrates that PNPLA3 influences liver fat accumulation early in life in Hispanic children and adults. ${ }^{34}$ This analysis also represents, individuals carrying the GG genotype of the PNPLA3 gene are susceptible to increased hepatic fat when dietary sugar intake is high. The role of PNPLA gene may have an association in the development of NAFLD-fib and NASH-cirrhosis in the Hispanics. Other studies have also confirmed that this gene predispose obese children and adolescents to exhibit hepatic damage. ${ }^{35}$

Among non-metabolic risk factors, $\mathrm{HCV}$ has long been recognized as a major predictor of $\mathrm{HCC}$ risk. ${ }^{36}$ This study is also consistent with previous studies indicating a largest risk of $\mathrm{HCV}$ in the non-Hispanic Black population ${ }^{36-37}$ A crosssectional study utilizing Medicare databases have shown that the proportion of HCC cases attributable to $\mathrm{HCV}$ and $\mathrm{HBV}$ have doubled over approximately the same study period. ${ }^{37,38}$ The differing estimations in previous studies compared to the current is likely due to the broader age of subjects surveyed presently in NHANES compared to SEER-Medicare databases. The known birth cohort effect of those born between 1945-1965 reaching the age of peak HCC risk had been previously reported, ${ }^{37,39}$ and the greater proportion of $\mathrm{HCV}$ contribution was towards HCC among the older US generation.

The current study does have certain limitations. While this study has assumed independent causation of $\mathrm{HCC}$ for each risk factor, the course of disease from obesity, diabetes, NAFLD-fib, and NASH-cirrhosis is not a mutually exclusive path toward malignancy. ${ }^{25,26,40-}$ ${ }^{43}$ Patients can often present with multiple risk factors, raising the question of how to accurately weigh the contribution of each risk and handle overlapping interactions with other, possibly concurrent, risk factors. While this study served to focus on contributions of each specific risk factor, 
future studies are needed to disentangle these interactions using real-world data.$^{44}$ Importantly, the average age of HCC onset is 65 while the study's cohort had an average age of 46 . While this study did adjust for age, the burden of each metabolic risk factor may have been an underestimation ${ }^{16}$ This study also assumes that people do not change their lifestyle habits as they grow older, thereby potentially decreasing their HCC risk ${ }^{29}$ Thus, these results should be interpreted as epidemiological evidence for prevention strategies and public health education on risk factors.

In conclusion, these results display the changing contributions as well as the proportions of known HCC risk factors among specific racial and ethnic groups in a representative sample of the US population. The results of this study show that the increasing $\mathrm{HCC}$ rates are due to modifiable causes; this can be used to inform prevention and education programs with awareness as to racial and ethnic genetic and lifestyle differences.

\section{References}

1. Shariff, M. I. F., Cox, I. J., Gomaa, A. I., Khan, S. A., Gedroyc, W., \& TaylorRobinson, S. D. (2009). Hepatocellular carcinoma: Current trends in worldwide epidemiology, risk factors, diagnosis and therapeutics. Expert Review of Gastroenterology and Hepatology.

https://doi.org/10.1586/egh.09.35

2. Parkin, D. M. (2001). Global cancer statistics in the year 2000. Lancet Oncology. https://doi.org/10.1016/S14702045(01)00486-7

3. Rinella, M. E. (2015). Nonalcoholic fatty liver disease a systematic review. JAMA - Journal of the American Medical Association. https://doi.org/10.1001/jama.2015.5370
4. Paschos, P., \& Paletas, K. (2009). Non alcoholic fatty liver disease and metabolic syndrome. Hippokratia.

5. Carrion, A. F., Ghanta, R., Carrasquillo, O., \& Martin, P. (2011). Chronic liver disease in the hispanic population of the united states. Clinical Gastroenterology and Hepatology. https://doi.org/10.1016/j.cgh.2011.04.027

6. Caetano, R., \& Kaskutas, L. A. (1995). Changes in drinking patterns among whites, blacks and Hispanics, 1984- 1992. Journal of Studies on Alcohol. https://doi.org/10.15288/jsa.1995.56.558

7. Nguyen, G. C., \& Thuluvath, P. J. (2008). Racial disparity in liver disease: Biological, cultural, or socioeconomic factors. Hepatology. https://doi.org/10.1002/hep.22223

8. Kulik, L., \& El-Serag, H. B. (2019). Epidemiology and Management of Hepatocellular Carcinoma. Gastroenterology. https://doi.org/10.1053/j.gastro.2018.08.065

9. Islami, F., Miller, K. D., Siegel, R. L., Fedewa, S. A., Ward, E. M., \& Jemal, A. (2017). Disparities in liver cancer occurrence in the United States by race/ethnicity and state. CA: A Cancer Journal for Clinicians. https://doi.org/10.3322/caac.21402

10. Rich, N. E., Hester, C., Odewole, M., Murphy, C. C., Parikh, N. D., Marrero, J. A., Yopp, A. C., \& Singal, A. G. (2019). Racial and Ethnic Differences in Presentation and Outcomes of Hepatocellular Carcinoma. Clinical Gastroenterology and Hepatology. https://doi.org/10.1016/j.cgh.2018.05.039

11. Prevention CfDCa. National Health and Nutrition Examination Survey Data. US Department of Health and Human Services. 2019.

12. (NCHS). National Health and Nutrition Examination Survey Analytic and Reporting Guidelines.: US Department of Health and 
Human Services, Centers for Disease Control and Prevention: Hyattsville, MD.

13. What's at-risk or heavy drinking? - Rethinking Drinking - NIAAA: National Institute on Alcohol Abuse and Alcoholism; [Available from:

https://www.rethinkingdrinking.niaaa.nih.gov/ How-much-is-too-much/Is-your-drinkingpattern-risky/Whats-At-Risk-Or-HeavyDrinking.aspx.

14. Alberti KG, Eckel RH, Grundy SM, Zimmet PZ, Cleeman JI, Donato KA, et al. (2009). Harmonizing the metabolic syndrome: a joint interim statement of the International Diabetes Federation Task Force on Epidemiology and Prevention; National Heart, Lung, and Blood Institute; American Heart Association; World Heart Federation; International Atherosclerosis Society; and International Association for the Study of Obesity. Circulation. 120(16):1640-5.

15. Lee JH, Kim D, Kim HJ, Lee CH, Yang JI, Kim W, et al. (2010). Hepatic steatosis index: a simple screening tool reflecting nonalcoholic fatty liver disease. Dig Liver Dis.; 42(7):503-8.

16. McPherson S, Stewart SF, Henderson E, Burt AD, Day CP. (2010). Simple non-invasive fibrosis scoring systems can reliably exclude advanced fibrosis in patients with nonalcoholic fatty liver disease. Gut.; 59(9):1265-9

17. Klein RJ SC. (2001). Age adjustment using the 2000 projected U.S. population. Healthy People Statistics Notes; 20.

18. Levin ML. (1953). The occurrence of lung cancer in man. Acta Unio Int Contra Cancrum.; 9(3):531-12.

19. Ong JP, Elariny H, Collantes R, Younoszai A, Chandhoke V, Reines HD, et al. (2005).

Predictors of nonalcoholic steatohepatitis and advanced fibrosis in morbidly obese patients. Obes Surg.; 15(3):310-5.
20. Piñero, J., Queralt-Rosinach, N., Bravo, À., Deu-Pons, J., Bauer-Mehren, A., Baron, M., Sanz, F., \& Furlong, L. I. (2015).

DisGeNET: A discovery platform for the dynamical exploration of human diseases and their genes. Database.

https://doi.org/10.1093/database/bav028

21. Shannon, P., Markiel, A., Ozier, O., Baliga, N. S., Wang, J. T., Ramage, D., Amin, N., Schwikowski, B., \& Ideker, T. (2003). Cytoscape: A software Environment for integrated models of biomolecular interaction networks. Genome Research. https://doi.org/10.1101/gr.1239303

22. Hassan, M. M., Kaseb, A., Etzel, C. J., ElSerag, H., Spitz, M. R., Chang, P., Hale, K. S., Liu, M., Rashid, A., Shama, M., Abbruzzese, J. L., Loyer, E. M., Kaur, H., Hassabo, H. M., Vauthey, J. N., Wray, C. J., Hassan, B. S., Patt, Y. Z., Hawk, E., ... Li, D. (2013). Genetic variation in the PNPLA3 gene and hepatocellular carcinoma in USA: Risk and prognosis prediction. Molecular Carcinogenesis. https://doi.org/10.1002/mc.22057

23. Ong JP, Elariny H, Collantes R, Younoszai A, Chandhoke V, Reines HD, et al. (2005). Predictors of nonalcoholic steatohepatitis and advanced fibrosis in morbidly obese patients. Obes Surg. ; 15(3):310-5.

24. Yasui K, Hashimoto E, Komorizono Y, Koike K, Arii S, Imai Y, et al. (2011). Characteristics of patients with nonalcoholic steatohepatitis who develop hepatocellular carcinoma. Clin Gastroenterol Hepatol.; 9(5):428-33; quiz e50.

25. Cholankeril G, Patel R, Khurana S, Satapathy SK. (2017). Hepatocellular carcinoma in nonalcoholic steatohepatitis: Current knowledge and implications for management. World J Hepatol.; 9(11):533 


\section{https://doi.org/10.48091/LKML8578}

26. Dongiovanni P, Romeo S, Valenti L. (2014). Hepatocellular carcinoma in nonalcoholic fatty liver: role of environmental and genetic factors. World J Gastroenterol.; 20(36):12945-55

27. Dhanasekaran R, Limaye A, Cabrera R. (2012). Hepatocellular carcinoma: current trends in worldwide epidemiology, risk factors, diagnosis, and therapeutics. Hepat Med. 4:1937.

28. Tampi RP, Wong VW, Wong GL, Shu SS, Chan HL, Fung J, et al. (2020). Modelling the Economic and Clinical Burden of NonAlcoholic Steatohepatitis in East Asia: Data from Hong Kong. Hepatol Res.

29. Tian Y, Li T, Qi S, Alhourani H, Luo B, Chenqin J, et al. (2020). The impact of metabolic syndrome (MetS) on surgical outcome for patients with mostly HBV-related hepatocellular carcinoma (HCC) underwent hepatectomy. J Surg Oncol.

30. McLaren L. (2007). Socioeconomic status and obesity. Epidemiol Rev.; 29:29-48.

31. Santoro, N., Kursawe, R., D’Adamo, E., Dykas, D. J., Zhang, C. K., Bale, A. E., Calí, A. M., Narayan, D., Shaw, M. M., Pierpont, B., Savoye, M., Lartaud, D., Eldrich, S., Cushman, S. W., Zhao, H., Shulman, G. I., \& Caprio, S. (2010). A common variant in the patatin-like phospholipase 3 gene (PNPLA3) is associated with fatty liver disease in obese children and adolescents. Hepatology (Baltimore, $M d$.). https://doi.org/10.1002/hep.23832

32. Romeo S, Kozlitina J, Xing C, Pertsemlidis A, Cox D, Pennacchio LA, et al. (2008). Genetic variation in PNPLA3 confers susceptibility to nonalcoholic fatty liver disease. Nat Genet. 40(12):1461-5.

33. Goran MI, Walker R, Le KA, Mahurkar S, Vikman S, Davis JN, et al. (2010). Effects of PNPLA3 on liver fat and metabolic profile in
Hispanic children and adolescents. Diabetes.; 59(12):3127-30.

34. Martinez LA, Larrieta E, Kershenobich D, Torre A. (2017). The Expression of PNPLA3 Polymorphism could be the Key for Severe Liver Disease in NAFLD in Hispanic Population. Ann Hepatol.; 16(6):909-15.

35. Altekruse SF, McGlynn KA, Reichman ME. (2009). Hepatocellular carcinoma incidence, mortality, and survival trends in the United States from 1975 to 2005. J Clin Oncol.; 27(9):1485-91.

36. Ott JJ, Stevens GA, Groeger J, Wiersma ST. (2012). Global epidemiology of hepatitis B virus infection: new estimates of age-specific $\mathrm{HBsAg}$ seroprevalence and endemicity. Vaccine.; 30(12):2212-9.

37. Degos F, Christidis C, Ganne-Carrie N, Farmachidi JP, Degott C, Guettier C, et al. (2000). Hepatitis $C$ virus related cirrhosis: time to occurrence of hepatocellular carcinoma and death. Gut.; 47(1):131-6.

38. Rosenberg ES, Rosenthal EM, Hall EW, Barker L, Hofmeister MG, Sullivan PS, et al. Prevalence of Hepatitis C Virus Infection in US States and the District of Columbia, 2013 to 2016. (2018). JAMA Netw Open.;1(8):e186371.

39. Denniston MM, Jiles RB, Drobeniuc J, Klevens RM, Ward JW, McQuillan GM, et al. (2014). Chronic hepatitis $C$ virus infection in the United States, National Health and Nutrition Examination Survey 2003 to 2010. Ann Intern Med.; 160(5):293-300.

40. Harris AM, Iqbal K, Schillie S, Britton J, Kainer MA, Tressler S, et al. Increases in Acute Hepatitis B Virus Infections Kentucky, Tennessee, and West Virginia, 2006-2013. (2016). MMWR Morb Mortal Wkly Rep.; 65(3):47-50.

41. Pham C, Fong TL, Zhang J, Liu L. Striking Racial/Ethnic Disparities in Liver Cancer 
https://doi.org/10.48091/LKML8578

Incidence Rates and Temporal Trends in California, 1988-2012. (2018). J Natl Cancer Inst.; 110(11):1259-69.

42. Le MH, Yeo YH, Cheung R, Henry L, Lok AS, Nguyen MH. (2020). Chronic Hepatitis B Prevalence Among Foreign-Born and U.S.Born Adults in the United States, 1999-2016. Hepatology.; 71(2):431-43.

43. Bugianesi E. (2005). Review article: steatosis, the metabolic syndrome and cancer. Aliment Pharmacol Ther.; 22 Suppl 2:40-3.

44. Bugianesi E, Leone N, Vanni E, Marchesini G, Brunello F, Carucci P, et al. (2002). Expanding the natural history of nonalcoholic steatohepatitis: from cryptogenic cirrhosis to hepatocellular carcinoma. Gastroenterology.;123(1):134-40.

45. Brumback B, Berg A. (2008). On effectmeasure modification: Relationships among changes in the relative risk, odds ratio, and risk difference. Stat Med.; 27(18):3453-65. 


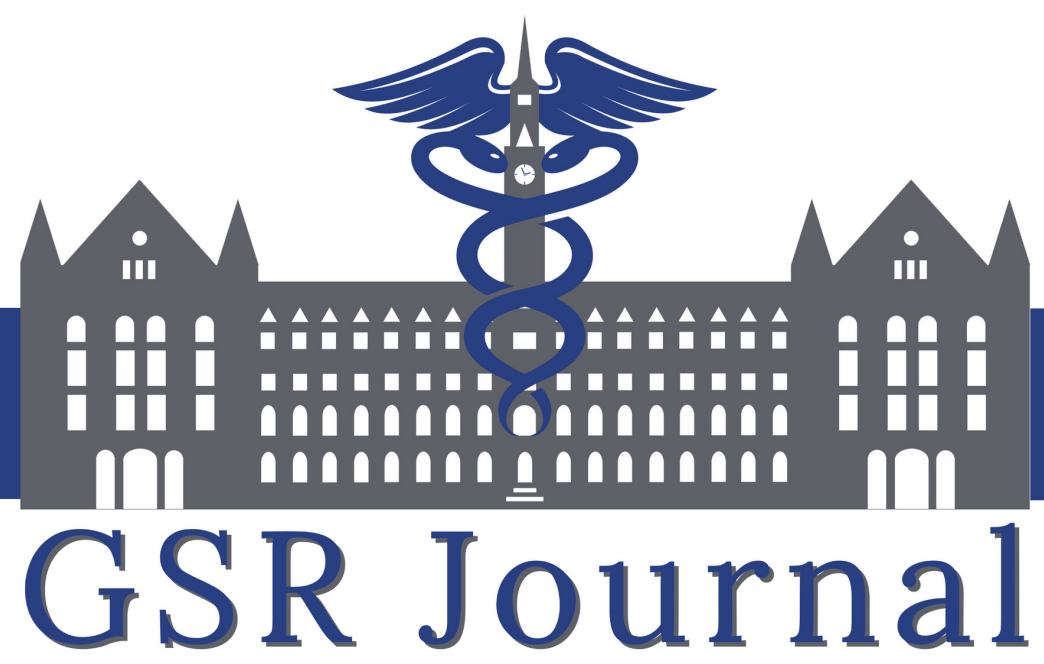

Georgetown Scientific Research Journal 